Research Article

\title{
Global Mittag-Leffler Stabilization of Fractional-Order BAM Neural Networks with Linear State Feedback Controllers
}

\author{
Hongyun Yan, ${ }^{1}$ Yuanhua Qiao $\left(\mathbb{D},{ }^{1}\right.$ Lijuan Duan, ${ }^{2}$ and Ling Zhang ${ }^{1}$ \\ ${ }^{1}$ Faculty of Sciences, Beijing University of Technology, Beijing 100124, China \\ ${ }^{2}$ Faculty of Information Technology, Beijing University of Technology, Beijing 100124, China \\ Correspondence should be addressed to Yuanhua Qiao; qiaoyuanhua@bjut.edu.cn
}

Received 11 December 2019; Revised 24 June 2020; Accepted 28 July 2020; Published 17 August 2020

Academic Editor: Jerzy Baranowski

Copyright (C) 2020 Hongyun Yan et al. This is an open access article distributed under the Creative Commons Attribution License, which permits unrestricted use, distribution, and reproduction in any medium, provided the original work is properly cited.

In this paper, the global Mittag-Leffler stabilization of fractional-order BAM neural networks is investigated. First, a new lemma is proposed by using basic inequality to broaden the selection of Lyapunov function. Second, linear state feedback control strategies are designed to induce the stability of fractional-order BAM neural networks. Third, based on constructed Lyapunov function, generalized Gronwall-like inequality, and control strategies, several sufficient conditions for the global Mittag-Leffler stabilization of fractional-order BAM neural networks are established. Finally, a numerical simulation is given to demonstrate the effectiveness of our theoretical results.

\section{Introduction}

Bidirectional associative memory (BAM) neural networks are a type of extended unidirectional auto-associator of Hopfield neural networks. They are composed of two layers: the $X$-layer and the $Y$-layer, which can store and recall pattern pairs [1]. And they are widely applied in pattern recognition, signal processing, and associative memories. It is of great importance to investigate the dynamic stability behaviors of such networks to meet application requirements.

The fractional-order model is developed quickly because of its memory and genetic characteristics [2-5]. When the fractional order is in the interval $[0,1]$, a new lemma for the Caputo fractional derivatives is proposed in [2]. Some properties of the Lyapunov direct method for noninteger order systems are presented in [3]. In [4], the authors extend Lyapunov direct method for noninteger order systems. In [5], the finite-time stability of fractional-order impulsive switched systems is considered. There are many research studies on fractional-order BAM neural networks [6-11]. A novel result about finite-time impulsive stability of fractional-order memristive BAM neural networks is obtained in [6]. Quasi-pinning synchronization and $\beta$-exponential pinning stabilization for a class of fractional-order BAM neural networks with time-varying delays and discontinuous neuron activations are considered in [8]. In [10], sufficient conditions for the existence, uniqueness, and global Mittag-Leffler stability for the solutions of the fractional difference model of BAM neural networks are provided. In [11], based on Cauchy-Schwartz inequality and Burkholder-Davis-Gundy inequality, some sufficient conditions are derived to ensure the uniform stability of stochastic fractional-order memristor fuzzy BAM neural networks.

The definitions of Mittag-Leffler stability and generalized Mittag-Leffler stability are proposed in [12, 13]. Subsequently, some investigations focus on Mittag-Leffler stability [14-21]. Global Mittag-Leffler stability and synchronization analysis of discrete fractional-order complexvalued neural networks with time delay are given in [14]. In [15], the global Mittag-Leffler stability of multiple equilibrium points for the impulsive fractional-order quaternionvalued neural networks is investigated by employing the Lyapunov method. In [16], sufficient conditions ensuring the existence, uniqueness, and global Mittag-Leffler stability of the solutions of the fractional-order coupled system on a network without strong connections are derived. In [17], a new criterion is proposed to ensure the Mittag-Leffler 
stability for fractional-order neural networks in the quaternion field. The finite-time Mittag-Leffler stability for fractional-order quaternion-valued memristive neural networks with impulsive effect is investigated in [20]. Researchers not only study stability but also introduce control strategies to improve the stability. Different controllers have been applied to postpone Hopf bifurcation and broaden the stability domain on fractional-order systems [22-25]. Adaptive control approaches are adopted to induce Mittag-Leffler stabilization and synchronization for delayed fractional-order BAM neural networks in [26]. With linear and partial state feedback controls, global Mittag-Leffler stability of fractional-order BAM neural network is analyzed using Caputo fractional derivative and generalized Gronwall inequality [27]. In [28], state feedback stabilizing control and output feedback stabilizing control are designed for the stabilization of fractional-order memristive neural networks. However, the influence of whole state feedback controllers on stability of fractional-order BAM neural networks is not considered.

Motivated by the above discussion, we investigate the global Mittag-Leffler stability of fractional-order BAM neural networks with linear feedback controllers, including single and whole state feedback controllers. The main contributions include the following: First, a novel lemma is proposed using basic inequality to broaden the choice of the Lyapunov function. Second, linear state feedback controllers are designed to stabilize the systems. Third, some sufficient conditions for global Mittag-Leffler stability are given by using the fractional Lyapunov method and introducing feedback controllers. Finally, numerical simulations are performed to show the effect of different state feedback controllers on the selected system.

The paper is organized as follows. The preliminaries and the model descriptions are given in Section 2. Some sufficient conditions for global Mittag-Leffler stability of fractional-order BAM neural networks with two types of feedback controls are given in Section 3. In Section 4, a numerical simulation using the Adams-type forecast correction method is presented to illustrate the effectiveness of the theoretical results. Conclusions are given in Section 5.

\section{Preliminaries and Model Description}

In this section, some relevant definitions and lemmas about fractional calculus are introduced and the fractional-order BAM neural networks are described. Caputo fractional derivative is adopted.

Definition 1 (see [27]). The Caputo fractional derivative of order $\alpha$ for a function $f(t) \in C^{n}([0,+\infty), R)$ is defined by

$$
{ }_{t}^{C} D_{0}^{\alpha} f(t)=\frac{1}{\Gamma(n-\alpha)} \int_{0}^{t}(t-s)^{n-\alpha-1} f^{(n)}(s) \mathrm{d} s,
$$

where $n-1<\alpha<n, n \in N_{+}, \Gamma(\alpha)$ is Euler's gamma function, and $\Gamma(\alpha)=\int_{0}^{+\infty} s^{\alpha-1} \exp (-s) \mathrm{d} t$.

Particularly, when $0<\alpha<1$,

$$
{ }_{t}^{C} D_{0}^{\alpha} f(t)=\frac{1}{\Gamma(1-\alpha)} \int_{0}^{t}(t-s)^{-\alpha} f^{\prime}(s) \mathrm{d} s .
$$

Definition 2 (see [27]). One-parameter Mittag-Leffler function is defined as

$$
E_{\alpha}(z)=\sum_{k=0}^{+\infty} \frac{z^{k}}{\Gamma(k \alpha+1)},
$$

and two-parameter Mittag-Leffler function is defined as

$$
E_{\alpha, \beta}(z)=\sum_{k=0}^{+\infty} \frac{z^{k}}{\Gamma(k \alpha+\beta)},
$$

where the real part $\operatorname{Re}(\alpha)$ of the complex number $\alpha$ is $\operatorname{Re}(\alpha)>0, z$ and $\beta$ are both complex numbers, and $\Gamma(\cdot)$ is Euler's gamma function. Obviously, $E_{\alpha}(z)=E_{\alpha, 1}(z)$, $E_{0,1}(z)=(1 /(1-z))$, and $E_{1,1}(z)=\exp (z)$.

In this paper, we consider the fractional-order BAM neural networks given by the following fractional differential equations:

$$
\left\{\begin{array}{l}
{ }_{t}^{C} D_{0}^{\alpha} x_{i}(t)=-a_{i} x_{i}(t)+\sum_{j=1}^{m} b_{i j} f_{j}\left(y_{j}(t)\right)+u_{i}(t), \quad t \geq 0, \\
{ }_{t}^{C} D_{0}^{\alpha} y_{j}(t)=-c_{j} y_{j}(t)+\sum_{i=1}^{n} d_{j i} g_{i}\left(x_{i}(t)\right)+v_{j}(t), \quad t \geq 0,
\end{array}\right.
$$

where ${ }_{t}^{C} D_{0}^{\alpha}$ is the Caputo derivative of order $0<\alpha<1$, $i=1,2, \ldots, n, j=1,2, \ldots, m, x_{i}$ and $y_{j}$ are the neural states, $a_{i}$ and $c_{j}>0$ are the self-inhibitions, $b_{i j}$ and $d_{j i}$ are the synaptic connection strengths, $f_{j}$ and $g_{i}$ are the activation functions satisfying $f_{j}(0)=0$ and $g_{i}(0)=0$, and $u_{i}(t)$ and $v_{j}(t)$ denote the external inputs.

To ensure the existence and uniqueness of solution of system (5), the following assumption is given.

Assumption 1. The neuron activation functions $f_{j}$ and $g_{i}$ satisfy Lipschitz condition with the Lipschitz constants $F_{j}>0$ and $G_{i}>0$, i.e., for $i \in\{1,2, \ldots, n\}$ and $j \in\{1,2, \ldots, m\}, \forall \theta_{1}, \theta_{2} \in R$,

$$
\begin{gathered}
\left|f_{j}\left(\theta_{1}\right)-f_{j}\left(\theta_{2}\right)\right| \leq F_{j}\left|\theta_{1}-\theta_{2}\right|, \\
\left|g_{i}\left(\theta_{1}\right)-g_{i}\left(\theta_{2}\right)\right| \leq G_{i}\left|\theta_{1}-\theta_{2}\right| .
\end{gathered}
$$

Now, we give the definition of the globally Mittag-Leffler stability for system (5) and some relevant lemmas.

Definition 3 (see [12]) (global Mittag-Leffler stability). Under the condition of $u_{i}(t)=0$ and $v_{j}(t)=0$ $(i=1,2, \ldots, n, j=1,2, \ldots, m)$, the zero solution of system (5) is globally Mittag-Leffler stable if there exist two positive constants $\eta_{1}, \eta_{2}>0$ such that for any trajectories $x(t)$ and $y(t)$ of system (5) with initial values $x_{0}$ and $y_{0}$, satisfying 


$$
\|x(t)\|_{p}+\|y(t)\|_{p} \leq\left\{\left(M_{1}\left(x_{0}\right)+M_{2}\left(y_{0}\right)\right) E_{\alpha}\left(-\eta_{1} t^{\alpha}\right)\right\}^{\eta_{2}}, \quad t \geq 0,
$$

where $\|\cdot\|_{p}$ is $p$-norm, $M_{1}\left(x_{0}\right) \geq 0, M_{2}\left(y_{0}\right) \geq 0, M_{1}(0)=0$, $M_{2}(0)=0$, and $E_{\alpha}(\cdot)$ is one-parameter Mittag-Leffler function.

Remark 1. If the equilibrium point is not at the origin, it can be shifted to the origin by coordinate transformation, so we just considered the case of zero equilibrium point.

Definition 4 (see [28]) (global Mittag-Leffler stabilization). System (5) is globally Mittag-Leffler stabilizable if there exists a suitable feedback control, such that closed-loop system (5) is globally Mittag-Leffler stable.

Lemma 1 (see [29]). If $x(t)=\left(x_{1}(t), x_{2}(t), \ldots\right.$, $\left.x_{n}(t)\right)^{T} \in R^{n}$ is a vector, where $x_{i}(t)$ are continuous and differentiable functions, for all $i=1,2, \ldots, n$, and $P \in R^{n \times n}$ is a positive definite matrix, then for general quadratic form function $x^{T}(t) P x(t)$, we have

$$
{ }_{t}^{C} D_{0}^{\alpha} x^{T}(t) P x(t) \leq 2 x^{T}(t) P_{t}^{C} D_{0}^{\alpha} x(t), \quad \forall \alpha \in(0,1) .
$$

Lemma 2 (see [27]) (generalized Gronwall-like inequality). Let $F(t)$ be a continuous function on $[0,+\infty)$, if there exist constants $\varrho_{1}>0$ and $\varrho_{2} \geq 0$ such that

$$
{ }_{t}^{C} D_{0}^{\alpha} F(t) \leq-\varrho_{1} F(t)+\varrho_{2}, \quad t \geq 0 .
$$

Then,

$$
F(t) \leq F(0) E_{\alpha}\left(-\varrho_{1} t^{\alpha}\right)+\varrho_{2} t^{\alpha} E_{\alpha, \alpha+1}\left(-\varrho_{1} t^{\alpha}\right), \quad t \geq 0,
$$

where $0<\alpha<1$ and $E_{\alpha}(\cdot)$ and $E_{\alpha, \alpha+1}(\cdot)$ are one-parameter Mittag-Leffler function and two-parameter Mittag-Leffler function, respectively.

Next, we propose a new lemma by applying basic inequality $a+b \geq 2 \sqrt{a b}, a, b \geq 0$ to broaden the choice of the Lyapunov function for analyzing the stability.

Lemma 3. For state vectors $x(t)=\left(x_{1}(t), x_{2}(t), \ldots, x_{n}(t)\right)^{T} \in R^{n} \quad$ and $y(t)=\left(y_{1}(t), y_{2}(t), \ldots, y_{m}(t)\right)^{T} \in R^{m}$, if there exist $\eta_{3}>0$ and two positive definite matrices $N_{n \times n}^{1}$ and $N_{m \times m}^{2}$, and all eigenvalues of $N^{1}$ and $N^{2}$ are greater than or equal to 1 , the following inequality is satisfied:

$$
\sum_{i=1}^{n} x_{i}^{2}(t)+\sum_{j=1}^{m} y_{j}^{2}(t) \leq\left[x(0)^{T} N^{1} x(0)+y(0)^{T} N^{2} y(0)\right] E_{\alpha}\left(-2 \eta_{3} t^{\alpha}\right) .
$$

Then,

$$
\left(\sum_{i=1}^{n} x_{i}^{2}(t)\right)^{1 / 2}+\left(\sum_{j=1}^{m} y_{j}^{2}(t)\right)^{1 / 2} \leq\left\{\left[2 x(0)^{T} N^{1} x(0)+2 y(0)^{T} N^{2} y(0)\right] E_{\alpha}\left(-2 \eta_{3} t^{\alpha}\right)\right\}^{1 / 2}
$$

where $0<\alpha<1, E_{\alpha}(\cdot)$ is one-parameter Mittag-Leffler function and $x(0)$ and $y(0)$ are the initial value.
Proof. By inequality (11) and basic inequality $a+b \geq 2 \sqrt{a b}$, we have

$$
\begin{aligned}
2\left(\sum_{i=1}^{n} x_{i}^{2}(t)\right)^{1 / 2} \times\left(\sum_{j=1}^{m} y_{j}^{2}(t)\right)^{1 / 2} & \leq \sum_{i=1}^{n} x_{i}^{2}(t)+\sum_{j=1}^{m} y_{j}^{2}(t) \\
& \leq\left[x(0)^{T} N^{1} x(0)+y(0)^{T} N^{2} y(0)\right] E_{\alpha}\left(-2 \eta_{3} t^{\alpha}\right) .
\end{aligned}
$$

Combining inequalities (11) and (13), we obtain

$$
\begin{aligned}
& {\left[\left(\sum_{i=1}^{n} x_{i}^{2}(t)\right)^{1 / 2}+\left(\sum_{j=1}^{m} y_{j}^{2}(t)\right)^{1 / 2}\right]^{2}} \\
& \quad=\sum_{i=1}^{n} x_{i}^{2}(t)+\sum_{j=1}^{m} y_{j}^{2}(t)+2\left(\sum_{i=1}^{n} x_{i}^{2}(t)\right)^{1 / 2} \times\left(\sum_{j=1}^{m} y_{j}^{2}(t)\right)^{1 / 2} \\
& \quad \leq\left[2 x(0)^{T} N^{1} x(0)+2 y(0)^{T} N^{2} y(0)\right] E_{\alpha}\left(-2 \eta_{3} t^{\alpha}\right) .
\end{aligned}
$$

Then,

$$
\begin{aligned}
& \left(\sum_{i=1}^{n} x_{i}^{2}(t)\right)^{1 / 2}+\left(\sum_{j=1}^{m} y_{j}^{2}(t)\right)^{1 / 2} \\
& \quad \leq\left\{\left[2 x(0)^{T} N^{1} x(0)+2 y(0)^{T} N^{2} y(0)\right] E_{\alpha}\left(-2 \eta_{3} t^{\alpha}\right)\right\}^{1 / 2} .
\end{aligned}
$$

\section{Main Results}

In this section, linear state feedback controls are designed and some sufficient conditions are derived to ensure global Mittag-Leffler stability of fractional-order BAM neural networks. 
3.1. A Single Linear State Feedback Control. The external inputs $u_{i}(t)$ and $v_{j}(t)$ in system (5), which only depend on a single linear state feedback control, are designed as follows:

$$
\begin{aligned}
& u_{i}(t)=h_{i} x_{i}(t), \\
& v_{j}(t)=k_{j} y_{j}(t) .
\end{aligned}
$$

Theorem 1. If

$$
H_{1}=\left(\begin{array}{cc}
A & \frac{C}{2} \\
\frac{C^{T}}{2} & B
\end{array}\right),
$$

is a $(n+m) \times(n+m)$ negative definite matrix, where $A_{n \times n}=$ $\operatorname{diag}\left\{-a_{i}+h_{i}\right\}$ and $B_{m \times m}=\operatorname{diag}\left\{-c_{j}+k_{j}\right\}$ are negative definite matrices and $C=\left(\left|b_{i j}\right| F_{j}+\left|d_{j i}\right| G_{i}\right)_{n \times m}$, then system (5) is globally Mittag-Leffler stable under designed control law (16).

Proof. Since $H_{1}$ is a negative definite matrix, there exist $0<l_{1 i}, \overline{l_{1 j}}<1$ such that $\bar{H}_{1}$ is a negative semidefinite matrix, where $\bar{A}=\operatorname{diag}\left\{\left(-a_{i}+h_{i}\right) l_{1 i}\right\}, \bar{B}=\operatorname{diag}\left\{\left(-c_{j}+k_{j}\right) \overline{l_{1 j}}\right\}$, and

$$
\bar{H}_{1}=\left(\begin{array}{cc}
\bar{A} & \frac{C}{2} \\
\frac{C^{T}}{2} & \bar{B}
\end{array}\right) .
$$

Consider a Lyapunov function as follows:

$$
V(t)=\sum_{i=1}^{n} x_{i}^{2}(t)+\sum_{j=1}^{m} y_{j}^{2}(t) .
$$

Using Lemma 1, we have

$$
\begin{aligned}
& { }_{t}^{C} D_{0}^{\alpha} V(t) \\
& \leq 2 \sum_{i=1}^{n} x_{i}(t)\left(-a_{i} x_{i}(t)+\sum_{j=1}^{m} b_{i j} f_{j}\left(y_{j}(t)\right)+h_{i} x_{i}(t)\right) \\
& +2 \sum_{j=1}^{m} y_{j}(t)\left(-c_{j} y_{j}(t)+\sum_{i=1}^{n} d_{j i} g_{i}\left(x_{i}(t)\right)+k_{j} y_{j}(t)\right) \\
& \leq 2 \sum_{i=1}^{n}\left(-a_{i} x_{i}^{2}(t)+\sum_{j=1}^{m}\left|b_{i j}\right|\left|f_{j}\left(y_{j}(t)\right)\right|\left|x_{i}(t)\right|+h_{i} x_{i}^{2}(t)\right) \\
& +2 \sum_{j=1}^{m}\left(-c_{j} y_{j}^{2}(t)+\sum_{i=1}^{n}\left|d_{j i}\left\|g_{i}\left(x_{i}(t)\right)\right\| y_{j}(t)\right|+k_{j} y_{j}^{2}(t)\right) \\
& \leq 2 \sum_{i=1}^{n}\left(\left(-a_{i}+h_{i}\right) x_{i}^{2}(t)+\sum_{j=1}^{m}\left|b_{i j}\right| F_{j}\left|y_{j}(t)\right|\left|x_{i}(t)\right|\right) \\
& +2 \sum_{j=1}^{m}\left(\left(-c_{j}+k_{j}\right) y_{j}^{2}(t)+\sum_{i=1}^{n}\left|d_{j i}\right| G_{i}\left|x_{i}(t)\right|\left|y_{j}(t)\right|\right) \\
& =2 \sum_{i=1}^{n}\left(-a_{i}+h_{i}\right) x_{i}^{2}(t)+2 \sum_{j=1}^{m}\left(-c_{j}+k_{j}\right) y_{j}^{2}(t) \\
& +2 \sum_{i=1}^{n} \sum_{j=1}^{m}\left(\left|b_{i j}\right| F_{j}+\left|d_{j i}\right| G_{i}\right)\left|x_{i}(t)\right|\left|y_{j}(t)\right| \\
& =2 \sum_{i=1}^{n}\left(-a_{i}+h_{i}\right) l_{1 i} x_{i}^{2}(t)+2 \sum_{j=1}^{m}\left(-c_{j}+k_{j}\right) \overline{l_{1 j}} y_{j}^{2}(t) \\
& +2 \sum_{i=1}^{n} \sum_{j=1}^{m}\left(\left|b_{i j}\right| F_{j}+\left|d_{j i}\right| G_{i}\right)\left|x_{i}(t)\right|\left|y_{j}(t)\right| \\
& +2 \sum_{i=1}^{n}\left(-a_{i}+h_{i}\right)\left(1-l_{1 i}\right) x_{i}^{2}(t)+2 \sum_{j=1}^{m}\left(-c_{j}+k_{j}\right)\left(1-\overline{l_{1 j}}\right) y_{j}^{2}(t) \\
& =2(|x(t)|,|y(t)|)^{T} \bar{H}_{1}(|x(t)|,|y(t)|) \\
& +2 \sum_{i=1}^{n}\left(-a_{i}+h_{i}\right)\left(1-l_{1 i}\right) x_{i}^{2}(t)+2 \sum_{j=1}^{m}\left(-c_{j}+k_{j}\right)\left(1-\overline{l_{1 j}}\right) y_{j}^{2}(t) \\
& \leq 2 \sum_{i=1}^{n}\left(-a_{i}+h_{i}\right)\left(1-l_{1 i}\right) x_{i}^{2}(t)+2 \sum_{j=1}^{m}\left(-c_{j}+k_{j}\right)\left(1-\overline{l_{1 j}}\right) y_{j}^{2}(t) .
\end{aligned}
$$




$$
\begin{aligned}
& \text { Let } \\
& \gamma_{1}=\min _{1 \leq i \leq n, 1 \leq j \leq m}\left\{\left(a_{i}-h_{i}\right)\left(1-l_{1 i}\right),\left(c_{j}-k_{j}\right)\left(1-\overline{l_{1 j}}\right)\right\} .
\end{aligned}
$$

According to the conditions of Theorem 1, obviously $\gamma_{1}>0$. Combining (20) and (21), we obtain

$$
{ }_{t}^{C} D_{0}^{\alpha} V(t) \leq-2 \gamma_{1} V(t) .
$$

Using Lemma 2, we obtain

$$
V(t) \leq V(0) E_{\alpha}\left(-2 \gamma_{1} t^{\alpha}\right)
$$

That is,

$$
\sum_{i=1}^{n} x_{i}^{2}(t)+\sum_{j=1}^{m} y_{j}^{2}(t) \leq\left[\sum_{i=1}^{n} x_{i}^{2}(0)+\sum_{j=1}^{m} y_{j}^{2}(0)\right] E_{\alpha}\left(-2 \gamma_{1} t^{\alpha}\right) \text {. }
$$

According to Lemma 3, we have

$$
\left(\sum_{i=1}^{n} x_{i}^{2}(t)\right)^{1 / 2}+\left(\sum_{j=1}^{m} y_{j}^{2}(t)\right)^{1 / 2} \leq\left\{\left[\sum_{i=1}^{n} 2 x_{i}^{2}(0)+\sum_{j=1}^{m} 2 y_{j}^{2}(0)\right] E_{\alpha}\left(-2 \gamma_{1} t^{\alpha}\right)\right\}^{1 / 2} .
$$

By Definitions 3 and 4, system (5) is globally Mittag-Leffler stable under designed control law (16).

3.2. The Whole Linear State Feedback Control. Meanwhile, we consider the following the external inputs $u_{i}(t)$ and $v_{j}(t)$ in system (5) that depend on the whole linear state feedback control:

$$
\begin{aligned}
& u_{i}(t)=\sum_{i=1}^{n} h_{i} x_{i}(t), \\
& v_{j}(t)=\sum_{j=1}^{m} k_{j} y_{j}(t) .
\end{aligned}
$$

In the proof of Theorem 1, it is a little strict to construct a negative definite matrix. Now, we will explore the stability of system (5) by establishing a nonzero matrix with zero diagonal elements and control law (26).

Theorem 2. If the conditions

$$
\begin{gathered}
\min _{1 \leq i \leq n, 1 \leq j \leq m}\left\{a_{i}-\frac{n h_{i}+\sum_{I=1}^{n} h_{I}}{2}-\lambda_{\max }\left(H_{2}\right), c_{j}\right. \\
\left.-\frac{m k_{j}+\sum_{J=1}^{m} k_{J}}{2}-\lambda_{\max }\left(H_{2}\right)\right\}>0,
\end{gathered}
$$

are satisfied and $\mathrm{H}_{2}$ is a $(n+m) \times(n+m)$ nonzero matrix with zero diagonal elements, where $C=\left(\left|b_{i j}\right| F_{j}+\left|d_{j i}\right| G_{i}\right)_{n \times m}$ and

$$
H_{2}=\left(\begin{array}{cc}
0 & \frac{C}{2} \\
\frac{C^{T}}{2} & 0
\end{array}\right) .
$$

$\lambda_{\max }\left\{\mathrm{H}_{2}\right\}$ is the largest eigenvalue of matrix $\mathrm{H}_{2}$; then, system (5) is globally Mittag-Leffler stable under designed control law (26).
Proof. Since the diagonal elements of $\mathrm{H}_{2}$ are 0 , the trace of $\mathrm{N}$ is 0 , and obviously, $\lambda_{\max }\left\{H_{2}\right\}>0$.

Consider the following Lyapunov function:

$$
V(t)=\sum_{i=1}^{n} x_{i}^{2}(t)+\sum_{j=1}^{m} y_{j}^{2}(t) .
$$

By Lemma 1, we obtain

$$
\begin{aligned}
{ }_{t}^{C} D_{0}^{\alpha} V(t) & \\
\leq & 2 \sum_{i=1}^{n} x_{i}(t)\left(-a_{i} x_{i}(t)+\sum_{j=1}^{m} b_{i j} f_{j}\left(y_{j}(t)\right)+\sum_{i=1}^{n} h_{i} x_{i}(t)\right) \\
& +2 \sum_{j=1}^{m} y_{j}(t)\left(-c_{j} y_{j}(t)+\sum_{i=1}^{n} d_{j i} g_{i}\left(x_{i}(t)\right)+\sum_{j=1}^{m} k_{j} y_{j}(t)\right) \\
= & 2 \sum_{i=1}^{n}\left(-a_{i} x_{i}^{2}(t)+\sum_{j=1}^{m} b_{i j} f_{j}\left(y_{j}(t)\right) x_{i}(t)+\sum_{I=1}^{n} h_{I} x_{I}(t) x_{i}(t)\right) \\
& +2 \sum_{j=1}^{m}\left(-c_{j} y_{j}^{2}(t)+\sum_{i=1}^{n} d_{j i} g_{i}\left(x_{i}(t)\right) y_{j}(t)+\sum_{J=1}^{m} k_{J} y_{J}(t) y_{j}(t)\right) \\
\leq & 2 \sum_{i=1}^{n}\left(-a_{i} x_{i}^{2}(t)+\sum_{j=1}^{m}\left|b_{i j}\right|\left|f_{j}\left(y_{j}(t)\right)\right|\left|x_{i}(t)\right|+\sum_{I=1}^{n} h_{I} x_{I}(t) x_{i}(t)\right) \\
& +2 \sum_{j=1}^{m}\left(-c_{j} y_{j}^{2}(t)+\sum_{i=1}^{n}\left|d_{j i}\right|\left|g_{i}\left(x_{i}(t)\right)\right|\left|y_{j}(t)\right|+\sum_{J=1}^{m} k_{J} y_{J}(t) y_{j}(t)\right) \\
\leq & 2 \sum_{i=1}^{n}\left(-a_{i} x_{i}^{2}(t)+\sum_{j=1}^{m}\left|b_{i j}\right| F_{j}\left|y_{j}(t)\right|\left|x_{i}(t)\right|+\sum_{I=1}^{n} h_{I} x_{I}(t) x_{i}(t)\right) \\
& +2 \sum_{j=1}^{m}\left(-c_{j} y_{j}^{2}(t)+\sum_{i=1}^{n}\left|d_{j i}\right| G_{i}\left|x_{i}(t)\right|\left|y_{j}(t)\right|+\sum_{J=1}^{m} k_{J} y_{J}(t) y_{j}(t)\right),
\end{aligned}
$$

due to

$$
\begin{aligned}
& x_{i}(t) x_{I}(t) \leq \frac{1}{2} x_{I}^{2}(t)+\frac{1}{2} x_{i}^{2}(t), \\
& y_{j}(t) y_{J}(t) \leq \frac{1}{2} y_{J}^{2}(t)+\frac{1}{2} y_{j}^{2}(t) .
\end{aligned}
$$

Substituting (31) into (30), we have 


$$
\begin{aligned}
& { }_{t}^{C} D_{0}^{\alpha} V(t) \leq 2 \sum_{i=1}^{n}\left(-a_{i}+\frac{n h_{i}+\sum_{I=1}^{n} h_{I}}{2}\right) x_{i}^{2}(t) \\
& +2 \sum_{j=1}^{m}\left(-c_{j}+\frac{m k_{j}+\sum_{J=1}^{m} k_{J}}{2}\right) y_{j}^{2}(t) \\
& +2 \sum_{j=1}^{m} \sum_{i=1}^{n}\left(\left|b_{i j}\right| F_{j}+\left|d_{j i}\right| G_{i}\right)\left|x_{i}(t)\right|\left|y_{j}(t)\right| \\
& =2 \sum_{i=1}^{n}\left(-a_{i}+\frac{n h_{i}+\sum_{I=1}^{n} h_{I}}{2}\right) x_{i}^{2}(t) \\
& +2 \sum_{j=1}^{m}\left(-c_{j}+\frac{m k_{j}+\sum_{J=1}^{m} k_{J}}{2}\right) y_{j}^{2}(t) \\
& +2(|x(t)|,|y(t)|)^{T} H_{2}(|x(t)|,|y(t)|) \\
& \leq 2 \sum_{i=1}^{n}\left(-a_{i}+\frac{n h_{i}+\sum_{I=1}^{n} h_{I}}{2}\right) x_{i}^{2}(t) \\
& +2 \sum_{j=1}^{m}\left(-c_{j}+\frac{m k_{j}+\sum_{J=1}^{m} k_{J}}{2}\right) y_{j}^{2}(t) \\
& +2 \lambda_{\max }\left(H_{2}\right)\left(\sum_{i=1}^{n} x_{i}^{2}(t)+\sum_{j=1}^{m} y_{j}^{2}(t)\right) \\
& =2 \sum_{i=1}^{n}\left(-a_{i}+\frac{n h_{i}+\sum_{I=1}^{n} h_{I}}{2}+\lambda_{\max }\left(H_{2}\right)\right) x_{i}^{2}(t) \\
& +2 \sum_{j=1}^{m}\left(-c_{j}+\frac{m k_{j}+\sum_{J=1}^{m} k_{J}}{2}+\lambda_{\max }\left(H_{2}\right)\right) y_{j}^{2}(t) \\
& \leq-2 \gamma_{2}\left(\sum_{i=1}^{n} x_{i}^{2}(t)+\sum_{j=1}^{m} y_{j}^{2}(t)\right)
\end{aligned}
$$

where

$$
\begin{aligned}
\gamma_{2}= & \min _{1 \leq i \leq n, 1 \leq j \leq m}\left\{a_{i}-\frac{n h_{i}+\sum_{I=1}^{n} h_{I}}{2}-\lambda_{\max }\left(H_{2}\right), c_{j}\right. \\
& \left.-\frac{m k_{j}+\sum_{J=1}^{m} k_{J}}{2}-\lambda_{\max }\left(H_{2}\right)\right\}>0 .
\end{aligned}
$$

That is,

$$
{ }_{t}^{C} D_{0}^{\alpha} V(t) \leq-2 \gamma_{2} V(t)
$$

Using Lemma 2, we obtain

$$
V(t) \leq V(0) E_{\alpha}\left(-2 \gamma_{2} t^{\alpha}\right)
$$

That is,

$$
\sum_{i=1}^{n} x_{i}^{2}(t)+\sum_{j=1}^{m} y_{j}^{2}(t) \leq\left[\sum_{i=1}^{n} x_{i}^{2}(0)+\sum_{j=1}^{m} y_{j}^{2}(0)\right] E_{\alpha}\left(-2 \gamma_{2} t^{\alpha}\right)
$$

According to Lemma 3, we have

$$
\begin{aligned}
& \left(\sum_{i=1}^{n} x_{i}^{2}(t)\right)^{1 / 2}+\left(\sum_{j=1}^{m} y_{j}^{2}(t)\right)^{1 / 2} \\
& \quad \leq\left\{\left[\sum_{i=1}^{n} 2 x_{i}^{2}(0)+\sum_{j=1}^{m} 2 y_{j}^{2}(0)\right] E_{\alpha}\left(-2 \gamma_{2} t^{\alpha}\right)\right\}^{1 / 2} .
\end{aligned}
$$

By Definitions 3 and 4, system (5) is globally Mittag-Leffler stable under designed control law (26).

Remark 2. In the proof of Theorems 1 and 2, by constructing a negative definite matrix or a nonzero matrix with zero diagonal elements, we enlarge the inequality and have ${ }_{t}^{C} D_{0}^{\alpha} V(t) \leq-2 \gamma_{i} V(t)$. Then, based on Lemma 2, the condition of Lemma 3 is satisfied $\sum_{i=1}^{n} x_{i}^{2}(t)+\sum_{j=1}^{m} y_{j}^{2}(t)$ $\leq\left[\sum_{i=1}^{n} x_{i}^{2}(0)+\sum_{j=1}^{m} y_{j}^{2}(0)\right] E_{\alpha}\left(-2 \gamma_{i} t^{\alpha}\right)$, where $N^{1}$ and $N^{2}$ of Lemma 3 are set to be unit matrices. Finally, using Lemma 3 and definition of Mittag-Leffler stability, we determine that system (5) is globally Mittag-Leffler stable under different control laws.

\section{Numerical Simulation}

In this section, a numerical example is given to show the effectiveness of our proposed theoretical results by Adama-Bashforth-Moulton predictor-corrector algorithm [30].

Consider the following four-dimensional fractional-order BAM neural networks:

$$
\left\{\begin{array}{l}
{ }_{t}^{C} D_{0}^{\alpha} x_{1}(t)=-1.2 x_{1}(t)+0.5 f_{1}\left(y_{1}(t)\right)+0.6 f_{2}\left(y_{2}(t)\right)+u_{1}(t), \\
{ }_{t}^{C} D_{0}^{\alpha} x_{2}(t)=-0.7 x_{2}(t)+0.8 f_{1}\left(y_{1}(t)\right)+0.2 f_{2}\left(y_{2}(t)\right)+u_{2}(t), \\
{ }_{t}^{C} D_{0}^{\alpha} y_{1}(t)=-0.85 y_{1}(t)+0.4 g_{1}\left(x_{1}(t)\right)+0.6 g_{2}\left(x_{2}(t)\right)+v_{1}(t), \\
{ }_{t}^{C} D_{0}^{\alpha} y_{2}(t)=-y_{2}(t)+0.1 g_{1}\left(x_{1}(t)\right)+0.3 g_{2}\left(x_{2}(t)\right)+v_{2}(t),
\end{array}\right.
$$

with the initial condition $\left(x_{1}(0), x_{2}(0), y_{1}(0), y_{2}(0)\right)^{T}=$ $(0.5,0.4,0.7,-0.1)^{T}$, where $\alpha=0.96, \quad f_{j}\left(y_{j}(t)\right)=(1 / 2)$ $\left(\left|y_{j}(t)+1\right|-\left|y_{j}(t)-1\right|\right), \quad$ and $\quad g_{i}\left(x_{i}(t)\right)=\tan h\left(x_{i}(t)\right)$, $i, j=1,2$.

As depicted in Figure 1, the state trajectories of system (38) without external controllers $u_{i}(t)$ and $v_{j}(t)$ cannot converge to the origin.

Based on selected activation functions $f_{j}$ and $g_{i}$, we have Lipschitz constants $F_{j}=G_{i}=1$. For single state feedback controller (16), setting $u_{1}(t)=-0.1 x_{1}(t), u_{2}(t)=-0.2 x_{2}(t)$, $v_{1}(t)=-0.1 y_{1}(t)$, and $v_{2}(t)=-0.2 y_{2}(t)$, we obtain a negative definite matrix $H_{1}$ : 


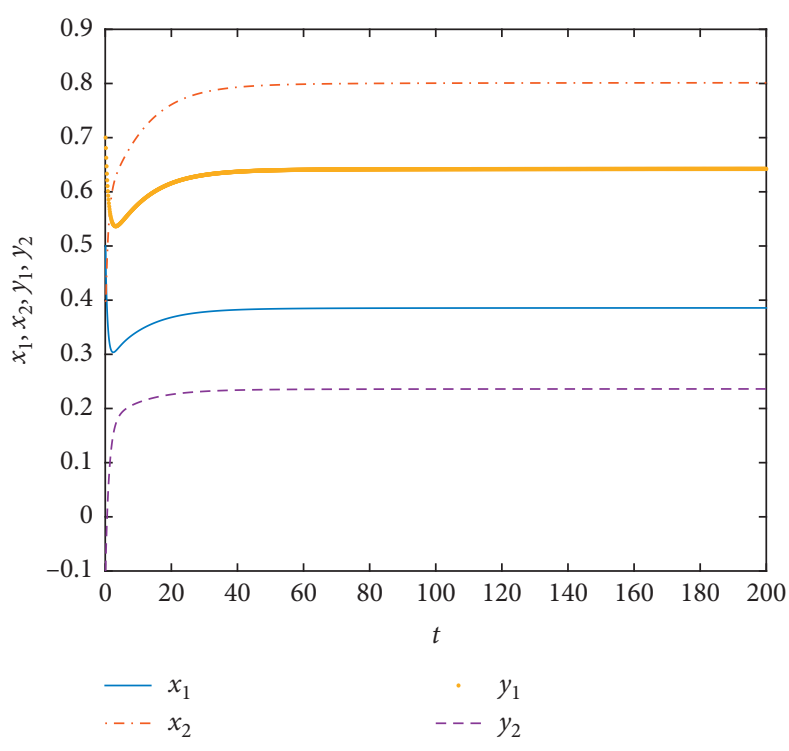

(a)

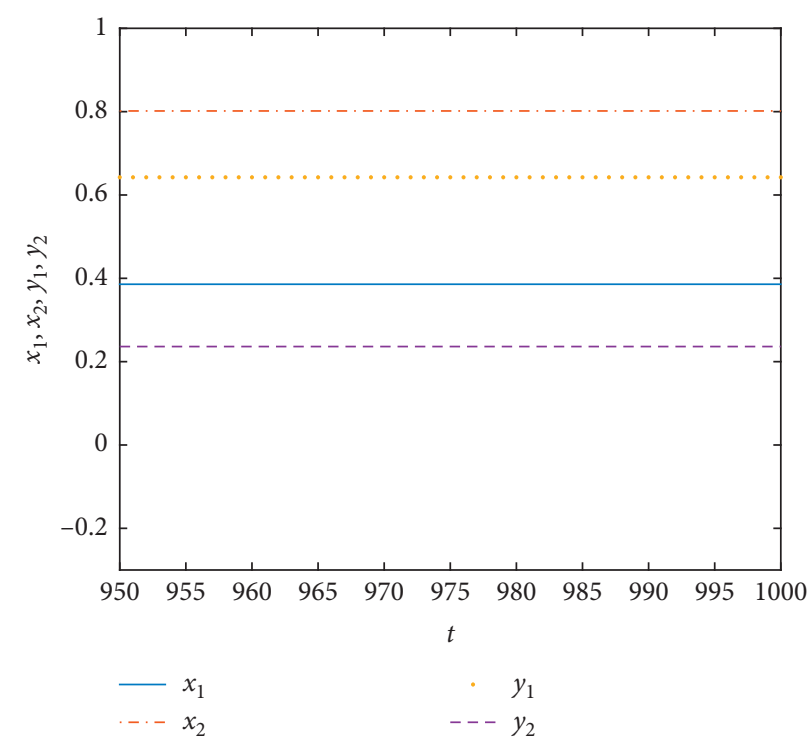

(b)

FIGURE 1: State trajectories of system (38) without external control.

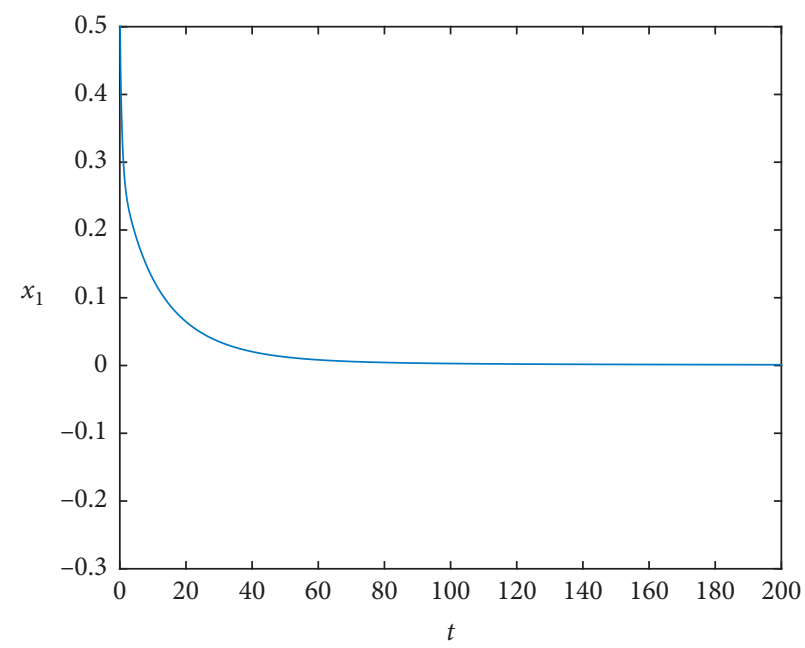

(a)

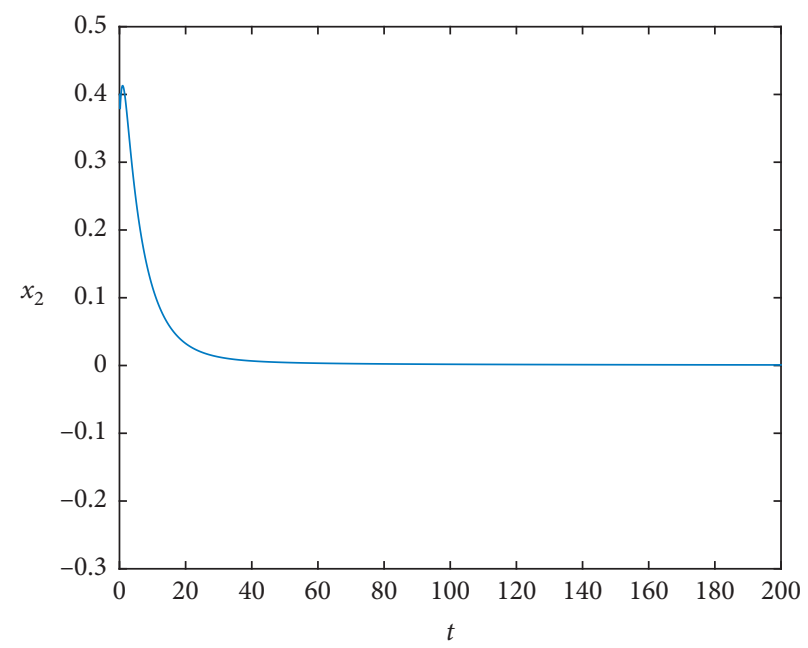

(b)

FIgURE 2: Continued. 


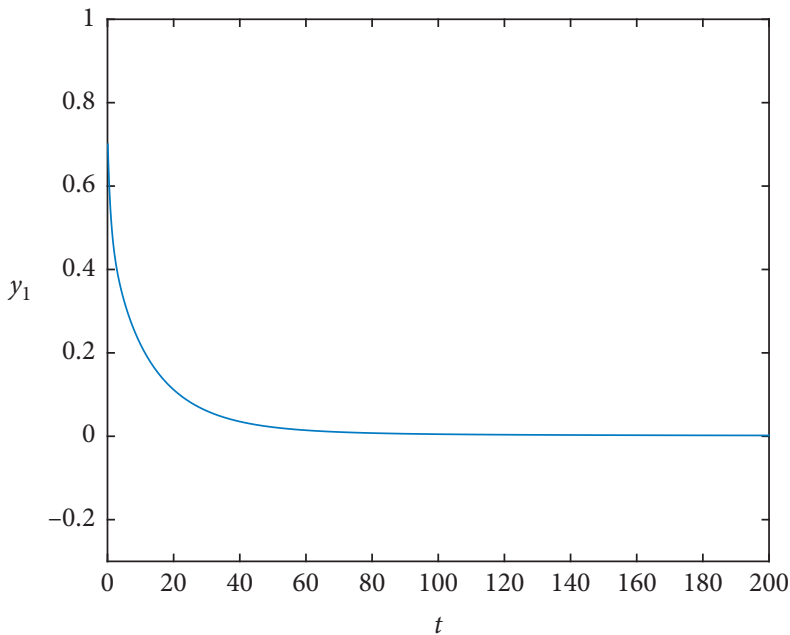

(c)

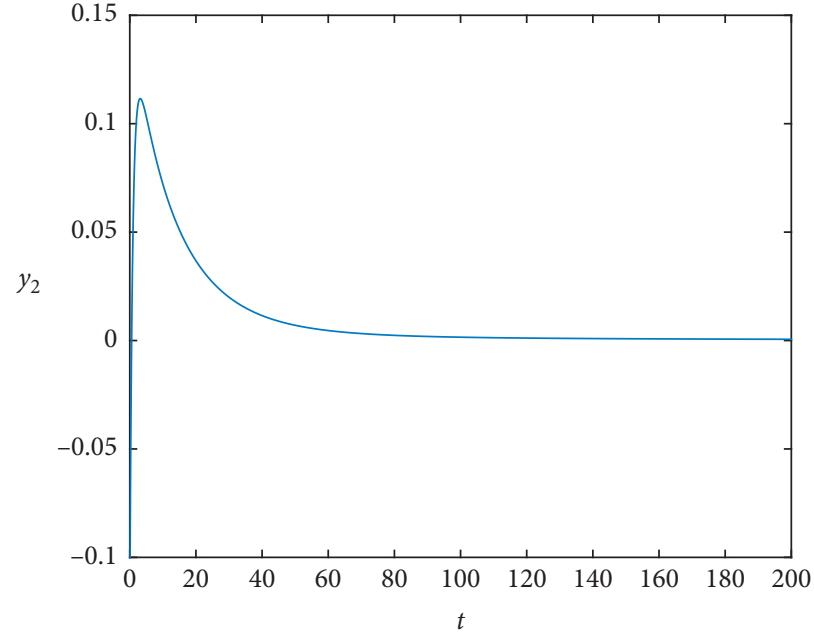

(d)

Figure 2: State trajectories of system (38) under the single state feedback laws $u_{1}(t)=-0.1 x_{1}(t), u_{2}(t)=-0.2 x_{2}(t), v_{1}(t)=-0.1 y_{1}(t)$, and $v_{2}(t)=-0.2 y_{2}(t)$.

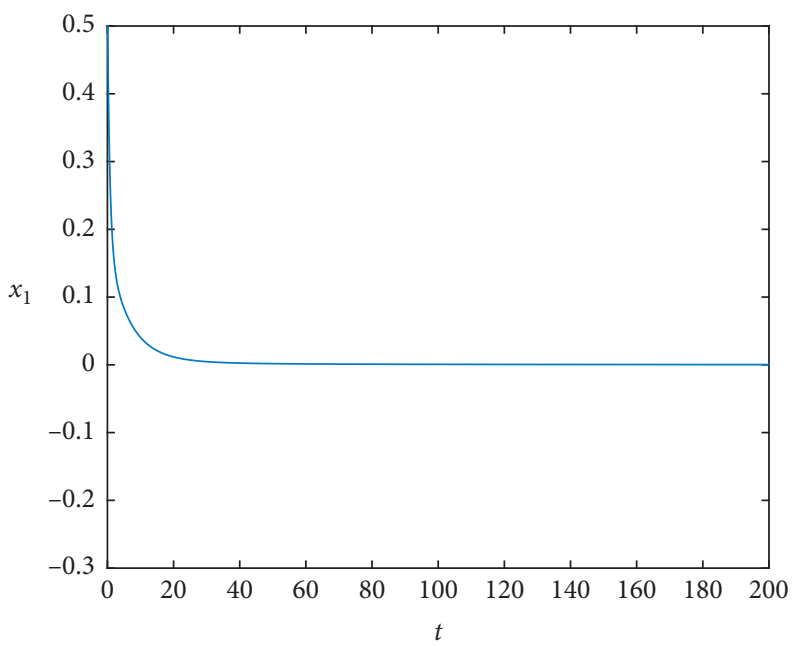

(a)

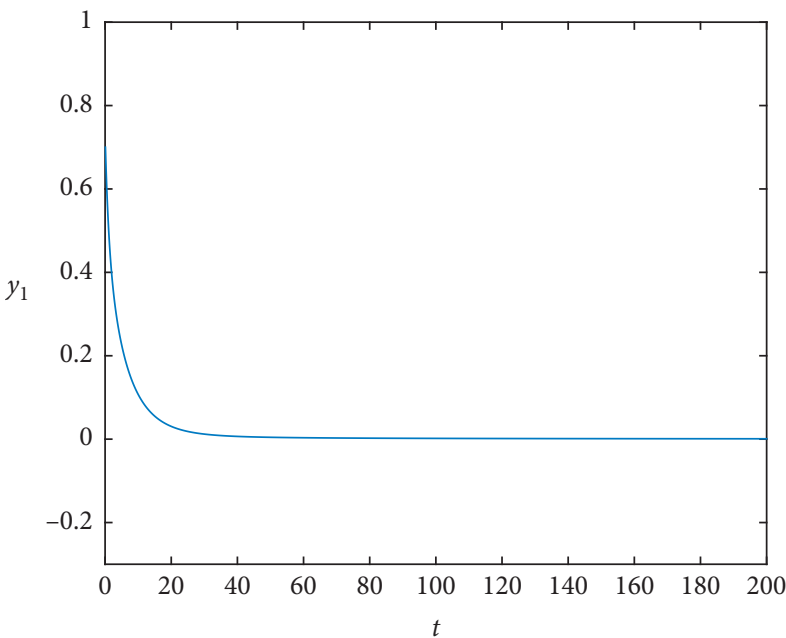

(c)

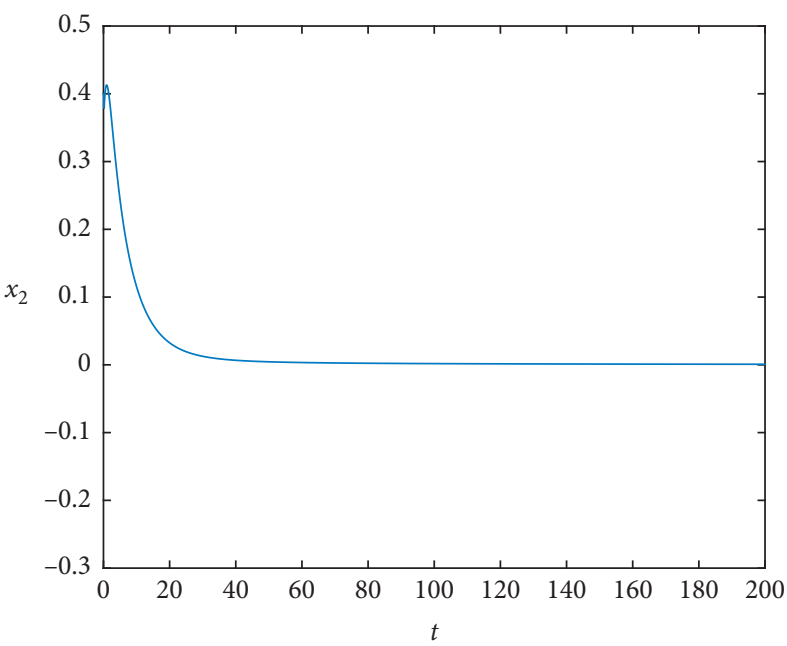

(b)

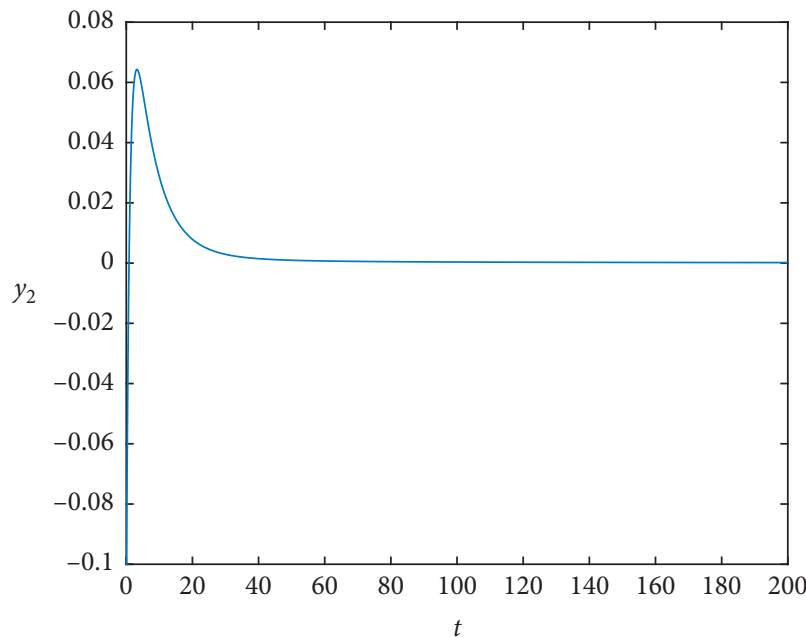

(d)

Figure 3: State trajectories of system (38) with the whole linear state feedback control laws $u_{1}(t)=u_{2}(t)=-0.2 x_{1}(t)-0.2 x_{2}(t)$ and $v_{1}(t)=v_{2}(t)=-0.1 y_{1}(t)-0.1 y_{2}(t)$. 


$$
H_{1}=\left(\begin{array}{cccc}
-1.3 & 0 & 0.45 & 0.35 \\
0 & -0.9 & 0.7 & 0.25 \\
0.45 & 0.7 & -0.95 & 0 \\
0.35 & 0.25 & 0 & -1.2
\end{array}\right) .
$$

Therefore, it follows from Theorem 1 that system (38) can achieve global Mittag-Leffler stability under the designed single linear state feedback control laws $u_{1}(t)=-0.1 x_{1}(t), \quad u_{2}(t)=-0.2 x_{2}(t), \quad v_{1}(t)=-0.1 y_{1}(t)$, and $v_{2}(t)=-0.2 y_{2}(t)$. Figure 2 shows state trajectories of system (38) with the designed single linear state feedback control law. As shown in Figure 2, state trajectories of system (38) converge to the origin.

By Theorem 2, we obtain a nonzero matrix with zero diagonal elements $\mathrm{H}_{2}$ and calculate the maximal eigenvalue of matrix $\lambda_{\max }\left(H_{2}\right)=0.9258$ :

$$
H_{2}=\left(\begin{array}{cccc}
0 & 0 & 0.45 & 0.35 \\
0 & 0 & 0.7 & 0.25 \\
0.45 & 0.7 & 0 & 0 \\
0.35 & 0.25 & 0 & 0
\end{array}\right) .
$$

For whole linear state feedback control law (26), let $u_{1}(t)=u_{2}(t)=-0.2 x_{1}(t)-0.2 x_{2}(t)$

and $v_{1}(t)=v_{2}(t)=-0.1 y_{1}(t)-0.1 y_{2}(t)$, the conditions of Theorem 2 are satisfied as follows:

$$
\left\{\begin{array}{l}
1.2-\frac{2 \times(-0.2)+(-0.2)+(-0.2)}{2}-\lambda_{\max }\left(H_{2}\right)>0, \\
0.7-\frac{2 \times(-0.2)+(-0.2)+(-0.2)}{2}-\lambda_{\max }\left(H_{2}\right)>0, \\
0.85-\frac{2 \times(-0.1)+(-0.1)+(-0.1)}{2}-\lambda_{\max }\left(H_{2}\right)>0, \\
1-\frac{2 \times(-0.1)+(-0.1)+(-0.1)}{2}-\lambda_{\max }\left(H_{2}\right)>0 .
\end{array}\right.
$$

Therefore, system (38) is globally Mittag-Leffler stable under the whole state feedback control law. Figure 3 shows state trajectories of system (38) with the designed whole linear state feedback control law.

\section{Conclusion}

In this paper, the global Mittag-Leffler stabilization problem for a class of fractional-order BAM neural networks is analyzed. Linear state feedback control is designed to induce the stability of fractional-order BAM neural networks, including a single linear state feedback control and the whole linear state feedback control. A novel lemma is proposed to broaden the selection of Lyapunov function. Some sufficient conditions of global Mittag-Leffler stability are given by using fractional Lyapunov stability theory, generalized Gronwall-like inequality, and the designed controls. In addition, a numerical example is given to show the influence of different state feedback controllers on the selected system. In the future research, we will try to investigate the effect of external disturbances on the stability of fractional-order BAM neural networks under linear state feedback controls. And we will explore in depth the stability of incommensurating fractional-order BAM neural networks with time delay.

\section{Data Availability}

The data used to support the findings of this study are included within the article.

\section{Conflicts of Interest}

The authors declare that they have no conflicts of interest.

\section{Acknowledgments}

This research was supported by the Beijing Municipal Natural Science Foundation (No. 4202025) and partially sponsored by the National Natural Science Foundation of China (No. 61672070) and Beijing Municipal Commission of Education (KZ201910005008).

\section{References}

[1] C. Huang and J. Cao, "Impact of leakage delay on bifurcation in high-order fractional BAM neural networks," Neural Networks, vol. 98, pp. 223-235, 2018.

[2] N. Aguila-Camacho, M. A. Duarte-Mermoud, and J. A. Gallegos, "Lyapunov functions for fractional order systems," Communications in Nonlinear Science and Numerical Simulation, vol. 19, no. 9, pp. 2951-2957, 2014.

[3] J. Baranowski, M. Zagorowska, W. Bauer et al., "Applications of direct Lyapunov method in Caputo non-integer order systems," Elektronika ir Elektrotechnika, vol. 21, no. 2, pp. 10-13, 2015.

[4] M. Zagórowska, J. Baranowski, W. Bauer, T. Dziwiński, P. Piątek, and W. Mitkowski, "Lyapunov direct method for non-integer order systems," Lecture Notes in Electrical Engineering, Springer, vol. 320, pp. 221-228, Berlin, Germany, 2015.

[5] Y. Yang and G. Chen, "Finite-time stability of fractional order impulsive switched systems," International Journal of Robust and Nonlinear Control, vol. 25, no. 13, pp. 2207-2222, 2015.

[6] L. Zhang and Y. Yang, "Finite time impulsive synchronization of fractional order memristive BAM neural networks," Neurocomputing, vol. 384, pp. 213-224, 2020.

[7] M. Syed Ali, G. Narayanan, V. Shekher, A. Alsaedi, and B. Ahmad, "Global Mittag-Leffler stability analysis of impulsive fractional-order complex-valued BAM neural networks with time varying delays," Communications in Nonlinear Science \& Numerical Simulation, vol. 83, Article ID 105088, 2020.

[8] A. Pratap, R. Raja, J. D. Cao, F. A. Rihan, and A. R. Seadawy, "Quasi-pinning synchronization and stabilization of fractional order BAM neural networks with delays and discontinuous neuron activations," Chaos, Solitons \& Fractals, vol. 131, Article ID 109491, 2020.

[9] A. Jehad, T. Swati, and A. Syed, "Discrete fractional-order BAM neural networks with leakage delay: existence and 
stability results," Asian Journal of Control, vol. 22, no. 1, pp. 143-155, 2020.

[10] A. Jehad, T. Swati, F. Ahmed et al., "On the stability and Lyapunov direct method for fractional difference model of BAM neural networks," Journal of Intelligent \& Fuzzy Systems, vol. 38, no. 3, pp. 2491-2501, 2020.

[11] M. Syed Ali, G. Narayanan, V. Shekher, H. Alsulami, and T. Saeed, "Dynamic stability analysis of stochastic fractionalorder memristor fuzzy BAM neural networks with delay and leakage terms," Applied Mathematics \& Computation, vol. 369, Article ID 124896, 2020.

[12] Y. Li, Y. Chen, and I. Podlubny, "Mittag-Leffler stability of fractional order nonlinear dynamic systems," Automatica, vol. 45, no. 8, pp. 1965-1969, 2009.

[13] Y. Li, Y. Chen, and I. Podlubny, "Stability of fractional-order nonlinear dynamic systems: Lyapunov direct method and generalized Mittag-Leffler stability," Computers \& Mathematics with Applications, vol. 59, no. 5, pp. 1810-1821, 2010.

[14] X. You, Q. Song, and Z. Zhao, "Global Mittag-Leffler stability and synchronization of discrete-time fractional-order complex-valued neural networks with time delay," Neural Networks, vol. 122, pp. 382-394, 2020.

[15] K. Udhayakumar, R. Rakkiyappan, J.-d. Cao, and X.-g. Tan, "Mittag-Leffler stability analysis of multiple equilibrium points in impulsive fractional-order quaternion-valued neural networks," Frontiers of Information Technology \& Electronic Engineering, vol. 21, no. 2, pp. 234-246, 2020.

[16] X. Meng, Y. G. Kao, H. R. Karimi et al., “Global Mittag-Leffler stability for fractional-order coupled systems on network without strong connectedness," Science China Information Sciences, vol. 63, no. 3, Article ID 132201, 2020.

[17] A. Pratap, R. Raja, J. Alzabut, J. Cao, G. Rajchakit, and C. Huang, "Mittag-Leffler stability and adaptive impulsive synchronization of fractional order neural networks in quaternion field," Mathematical Methods in the Applied Sciences, vol. 43, no. 10, pp. 6223-6253, 2020.

[18] H. Li and Y. Kao, "Mittag-Leffler stability for a new coupled system of fractional-order differential equations with impulses," Applied Mathematics and Computation, vol. 361, pp. 22-31, 2019.

[19] S. Ndolane, "Mittag-Leffler input stability of fractional differential equations and its applications," Discrete \& Continuous Dynamical Systems-Series S, vol. 13, no. 3, pp. 867-880, 2020.

[20] A. Pratap, R. Raja, J. Alzabut, J. Dianavinnarasi, J. Cao, and G. Rajchakit, "Finite-time Mittag-Leffler stability of fractional-order quaternion-valued memristive neural networks with impulses," Neural Processing Letters, vol. 51, no. 2, pp. 1485-1526, 2020.

[21] J. Deng, W. Ma, K. Deng, and Y. Li, “Tempered Mittag-Leffler stability of tempered fractional dynamical systems," Mathematical Problems in Engineering, vol. 2020, Article ID 7962542, 9 pages, 2020.

[22] X. Wang, Z. Wang, and J. Xia, "Stability and bifurcation control of a delayed fractional-order eco-epidemiological model with incommensurate orders," Journal of the Franklin Institute, vol. 356, no. 15, pp. 8278-8295, 2019.

[23] Q. Lu, M. Xiao, Z. Cheng, Y. Song, C. Huang, and J. Cao, "Stability and bifurcation analysis of a fractional-order singlegene regulatory model with delays under a novel $\mathrm{PD}^{\alpha}$ control law," International Journal of Biomathematics, vol. 13, no. 3, Article ID 2050016, 2020.

[24] S. Shi, M. Xiao, L. Rong et al., "Stability and bifurcation control of a neuron system under a novel fractional-order PD controller," Science China Technological Sciences, vol. 62, no. 12, pp. 2120-2129, 2019.

[25] C. Xu, M. Liao, and P. Li, "Bifurcation control of a fractionalorder delayed competition and cooperation model of two enterprises," Science China Technological Sciences, vol. 62, no. 12, pp. 2130-2143, 2019.

[26] W. K. Cheng, A. L. Wu, J. E. Zhang et al., "Adaptive control of Mittag-Leffler stabilization and synchronization for delayed fractional-order BAM neural networks," Advances in Difference Equations, vol. 2019, no. 1, 2019.

[27] A. Wu, Z. Zeng, and X. Song, "Global Mittag-Leffler stabilization of fractional-order bidirectional associative memory neural networks," Neurocomputing, vol. 177, pp. 489-496, 2016.

[28] A. L. Wu and Z. G. Zeng, "Global Mittag-Leffler stabilization of fractional-order memristive neural networks," IEEE Transactions on Neural Networks and Learning Systems, vol. 28, no. 1, pp. 206-217, 2015.

[29] H. Q. Wu, L. F. Wang, Y. Wang et al., "Global Mittag-Leffler projective synchronization for fractional-order neural networks: an LMI-based approach," Advances in Difference Equations, vol. 2016, no. 1, 2016.

[30] S. Bhalekar and V. Daftardar-Gejji, "A predictor-corrector scheme for solving nonlinear delay differential equations of fractional order," Journal of Fractional Calculus and Applications, vol. 1, no. 5, pp. 1-9, 2011. 